\title{
Weiteres iiber Quecksilberchlorid -Chlornatrium.
}

Von Julius Müller, Apotheker in Breslau.

(Vorgetragen in der Sehlesischen Gesellschaft für vaterländisehe Cultur;

Medicinische Section den 7. Juli 1871.)

Am 1. Juli vorigen Jahres empfahl ich in Gemeinschaft mit Herrn Dr. Stern das Quecksilberchlorid-Chlornatrium mit Ueberschuss von Chlornatrium zur therapeutischen Anwendung. - Heute veranlassen mich die mir vom Herrn Prof. Dr. Förster und Herrn Privatdocent Dr. Röbner mitgetheilten darüber gemachten Erfahrungen, sowie ein Aufsatz in der letzten Nummer des Archivs der Pharmacie darauf zurückzukommen. Herr C. Schering in Berlin, Besitzer einer der grössten chemischen Fabriken in Deutschland, empfiehlt darin das von ihm in Folge der von Herrn Dr. Stern und mir in dieser Zeitschrift veröffentlichten Abhandlung dargestellte Präparat "Hydrargyro - Natrium chloratum." Ehe ich näher darauf eingehe, will ich ganz kurz noch einmal die Gründe angeben, die mich namentlich bewogen, das Quecksilberchlorid-Chlornatrium mit überschüssigem Chlornatrium zu empfehlen.

Das metallische Quecksilber löst sich durch Schütteln mit Chlornatriumlösung in der erwähnten Form auf. Ausgrehend von dem chemischen Grundsatz "corpora non agunt, nisi soluta " zog ioh daraus den Schluss, dass bei der Schmierkur das Quecksilber durch den namentlich an Chlornatrium so reichen Schweiss zil dieser Verbindung gelöst und in dieser Form die antisyphilitische Wirkung ausibe. Ferner fand ich, dass alle Quecksilberprïparate mit einziger Ausnahme des absolut unlöslichen Schwefelquecksilbers durch Chlornatrium, also gewiss auch durch die an Chloriden so reichen thierischen Flüssigkeiten in einer Form gelöst werden, die Eiweiss nicht fällt, eine Eigenschaft, die eben das Quecksilberchlorid-Chlornatrium durch einen Zusatz von mindestens 10 Theilen Chlornatrium ebenfalls erlangt und die meiner Ansicht nach von grosser Wichtigkeit ist. Ich sagte damals, dass in dieser Form alles Quecksilberchlorid im Organismus, 
obne Störungen zu veranlassen, sofort zur Wirkung kommen miisse, wogegen bei Anwendung von Quecksilberchlorid ohne Chlornatrium sicher ein grosser Theil des Sublimats durch das Eiweiss des Organismus gefällt, also augenblicklich gewiss unwirksam gemacht und hierdurch die mannichfachen Störungen im Organismus beim Sublimatgebrauch veranlasst werden

Ich schlug ferner in Folge der im Ganzen gewiss sehr geringen Menge vermittels des Chlornatrium im Schweiss gelösten Quecksilbers bei Anwendung der Schmierkur vor, sehr kleine Mengen Sublimat in der erwähnten Verbindung zum innern Gebranch zu benutzen. -

Heut nach Ablauf eines Jahres gehört das Mittel glücklicher Weise noch nicht zu den vielen ad acta gelegten. Im hiesigen Allerheiligen-Hospital ist auf der syphilitischen Station das Mittel bis vor Kurzem ausschliesslich angewandt worden und zwar so, dass der Patient von einer Mixtur, die in $180 \mathrm{Grm}$. $6 \mathrm{Grm}$. Chlornatrium und $0,03 \mathrm{Grm}$. Sublimat enthielt, zweistündlich einen Esslöffel, also pro die 0,015 Sublimat bekam. Das Mittel wurde sehr gut rertragen, nur zeigte sich, dass die Kur langsamer als bei Anwendung der Schmierkur verlief; vor Allem war dies bei schweren Fällen zu constatiren.

Ich fühle mich in Folge dessen genöthigt, den einen gezogenen Schluss, dass abnorm kleine Mengen von Sublimat in der erwähnten Verbindung heilend wirken möchten, fallen zu lassen, kann aber wohl aufrecht halten, dass das Quecksilberchlorid in dieser Verbindung weit besser als reines Sublimat vertragen wird.

Die Herren Prof. Dr. Förster und Privatdocent Dr. Röbner, die die Freundlichkeit hatten, sich ebenfalls für das Mittel zu interessiren, hatten so günstige Erfahrungen, wie sie mir Herr Sanitätsrath Dr. Herdann aus dem Allerheiligen-Hospital mitgetheilt, nicht gemacht, erwähnten aber gleichzeitig, dass, da ja nach meinen Untersuchungen schon die zehnfache Menge des Chlornatriums genüge, um die Fällbarkeit des Eiweiss aufzuheben, sie eine bei Weitem geringere 
Menge Chlornatrium, wie sie in Hospital angewandt wurde, zugesetzt hätten. -

Die Erfahrungen standen sich also gewisser Maassen im Widerspruch; es musste dies sicher am Mittel selbst liegen und dies stellte sich in der That bald heraus. - Es ist richtig, dass, wenn man zu einem Theil in möglichst wenig Wasser gelösten Quecksilberchlorids zehn Theile Chlornatrium zufügt, Eiweisslösung von dieser Flüssigkeit nicht gefällt wird; verdünnt man aber solche Lösung mit viel Wasser, so erlangt diese so verdünnte Flüssigkeit wieder die Eigenschaft, die Eiweisslösung zu trüben. Ioh löste beispielsweise $1 \mathrm{Grm}$. Chlornatrium in $10 \mathrm{Grm}$. Wasser und fügte hierzu $0,1 \mathrm{Grm}$. Quecksilberchlorid. Diese concentrirte Lösung fällt Eiweisslösung nicht, verdünnte ich diese Flüssigkeit aber bis zu 180 Grm., so wurde davon Eiweiss getrubt; es ging also daraus hervor, dass in dieser Verdünnung die Menge des Chlornatrium nicht hinreichend war, dass also gewiss da, wo das Mittel dieselben unangenehmen Nebenwirkungen wie Sublimat ohne Chlornatrium erzeugt, die Menge des zugefügten Chlornatrium eine zu geringe gewesen sein mag. -

Als ich nach dieser Erfahrung in dem Archiv der Pharmacie den erwähnten Aufsatz von Schering las und fand, dass das von ihm dargestellte Hydrargyro-Natrium chloratum auf 1 Aequivalent Quecksilberchlorid nur 1 Aequivalent Chlornatrium, d. i. nach Gewichtstheilen noch nicht ganz die Hälfte enthielt, sagte ich mir bald, dass, da dieses Präparat sicher in Folge der geringen Menge Chlornatrium Eiweisslösung trüben würde, es dem von mir empfohlenen Mittel mehr schaden wie nützen müsse, d. h. dass es vor dem Gebrauch des reinen Sublimats keinerlei Vorzug haben könne. Eine von Schering bezogene Probo überzeugte mich von der Richtigkeit meiner Voraussetzung. Dieses Salz, gelöst in Wasser, trübt Eiweisslösung sofort, wogegen das von mir nach einer gleich zu erwähnenden Vorschrift bereitete Salz Eiweiss völlig klar lässt. Ja es verhält sich auch gegen chemische Reagentien ganz anders: wenn das Schering'sche Präparat mit dem geringen Chlornatriumgehalt bei Zusatz von Kalilauge einen 
gelbrothen Niederschlag giebt, so erzeugt Kalilauge in dem von mir dargestellten Salz einen weissen.

Es ist also die reine von Schering dargestellte chemische Verbindung von Quécksilberchlorid-Chlornatrium ohne vermehrten Zusatz von Chlornatrium mit meinem Vorschlage in keinem Zusammenhang zu bringen. Um nun den Aerzten ein - gewiss Vortheile vor dem reinen Sublimat bietendes Präparat zu liefern und eine leichte Verschreibweise zu ermöglichen, stellte ich das Hydrarg. bichlor. cum Natrio chlorato dar. Es enthält auf 1 Theil Quecksilberchlorid 100 Theile Chlornatrium, in jedem Gramme also $0,01 \mathrm{Grm}$. Sublimat und löst sich in wenig Wasser leicht auf. Man stellt es dar, indem man 100 Theile Chlornatrium und 1 Theil Quecksilberchlorid in destillirtem Wasser löst und die filtrirte Lösung bis zur Trockene eindampft. Im Allerheiligen-Hospital wird es seit Kurzem verordnet und zwar so, dass 6 Grm. des Salzes in 180 Grm. Wasser gelöst und hiervon der Patient zweistündlich einen Esslöffel erhält, pro die also 0,03 Grm. Sublimat nimmt. -

Ich bin überzeugt, dass es auch in dieser grösseren Dosis, wie es bisher angewandt, gut vertragen wird und hoffe, dass, da die längere Dauer der Kur sicher nur in der zu geringen Dosis lag, auch dieser Vorwurf fallen wird. Denn ist man überzeugt, dass bei der Schmierkur das Quecksilber, um die antisyphilitische Wirkung auszuüben, sich lösen muss, so kann es kaum in einer anderen als in der von mir erwähnten Form sein und es ist gewiss rationeller, das zur Heilung nöthige Quecksilber in der am leichtesten assimilirbaren Form und in der nur unbedingt erforderlichen Menge dem Organismus zuzufiuhren, als eine nicht zu bestimmende Menge Quecksilber vermittels der Schmierkur in den Körper za bringen. 